\title{
Introduction to Flap Movement: Reconstruction of Five Similar Nasal Defects Using Different Flaps
}

\author{
Elbert H. Chen, MD, ${ }^{*}$ Timothy M. Johnson, MD ${ }^{\dagger}$ And Désirée Ratner, MD* \\ *Department of Dermatology, Columbia University College of Physicians and Surgeons, New York, New York; \\ Departments of Dermatology, Otorhinolaryngology, and Surgery, University of Michigan Medical Center, Ann Arbor, \\ Michigan
}

BACKGROUND. There are several options for closure of a given surgical defect after tumor extirpation is confirmed. Flap reconstruction is one of these options.

OBJECTIVE. The purpose of this article is to introduce the three basic types of flap movement: advancement, rotation, and transposition.

METHODS. Five similar defects located on the nasal sidewall were repaired, each using a different flap design.
RESULTS. The optimal flap design for a given defect on a particular patient is based on the answers to a series of questions: Where is the available tissue reservoir? How can tissue be mobilized from the reservoir to cover the defect? How do the resulting tension vectors affect critical structures? Where are the final incision lines? CONCLUSION. Many factors must be evaluated before determining a method of reconstruction. Flap reconstruction requires a thorough understanding of anatomy and tissue movement.

ELBERT H. CHEN, MD, TIMOTHY M. JOHNSON, MD, AND DÉSIRÉE RATNER, MD, HAVE INDICATED NO SIGNIFICANT INTEREST WITH COMMERCIAL SUPPORTERS.

THE FUNDAMENTAL oncologic principle is tumor removal first, reconstruction second. Complete tumor extirpation may be confirmed by a variety of methods, including permanent section margin control, frozen-section margin control, or Mohs micrographic surgery. ${ }^{1}$ Once tumor clearance has been established, several factors must be evaluated before determining a method of reconstruction. Patient considerations include patient needs, general medical condition, and psychosocial factors. Defect considerations include the size, depth, and location of the defect. The location of the defect influences structure, function, and the availability of adjacent tissue reservoirs. Finally, the surgeon's experience and personal preferences play a role. The ideal closure provides the best esthetic and functional result while avoiding potential complications, accommodating the patient's needs, and incorporating the patient's ability to participate in postoperative care.

Alternatives for closure of a given surgical defect include primary closure, second-intention healing, skin grafting, or local tissue transfer with flaps. Primary or side-to-side closure is the simplest, most frequently used option and often provides the best result. Second-intention healing can give excellent functional and cosmetic results, particularly when used for defects located on concave sur-

Address correspondence and reprint requests to: Désirée Ratner, MD, Department of Dermatology, Columbia University Medical Center, 161 Fort Washington Avenue, 12th Floor, New York, NY 10032, or e-mail: dr221@columbia.edu. faces of the nose, eye, ear, and temple. ${ }^{2}$ Skin grafts have several disadvantages when compared with primary closure or a local flap; however, when properly executed, skin grafts can be successful both functionally and cosmetically. ${ }^{3}$

\section{Flaps}

A flap is a section of full-thickness skin that is incised leaving a proximal portion attached, thereby providing an intact vascular supply, whereas the distal portion is undermined and moved to another location to cover a defect. Flap reconstruction has several advantages over both primary closure and skin grafting. It allows repair with skin of similar characteristics, provides tissue bulk for reconstruction of the depth of a wound, and can also redirect or redistribute closure tension.

There are several methods to categorize flaps. ${ }^{4-6}$ The most useful relates to the predominant direction of tissue movement used to cover the defect. The advancement flap is so named because its primary tissue movement is entirely in one direction. The primary advantage of advancement flaps is that they allow redirection of incision lines and standing cones to more favorable sites. Rotation flaps rotate around a pivot point into a defect. Rotation flaps redirect and redistribute some tension from the primary defect to the secondary defect. Transposition flaps are lifted and rotated from the donor site over an intervening segment of intact skin and into the primary defect. 
The most important advantage of transposition flaps is that they can be designed to redirect nearly all of the tension from the primary to the secondary defect. In addition, compared with advancement and rotation flaps, transposition flaps generally have smaller but more numerous incisions. Most flaps incorporate more than one mode of tissue movement. An alternative classification of flaps may be defined based on two types of tissue movement: sliding and lifting. ${ }^{5,7}$ Flaps may also be grouped according to blood supply. The majority of flaps used in dermatologic surgery are random pattern flaps, which rely on either the surrounding reticular dermal vessels or perforating vessels from the subdermal plexus for their blood supply. In contrast, axial pattern flaps are designed to obtain their blood supply from a named artery.

\section{Case Examples}

Using a series of five similar defects located on the nose, each repaired with a different flap, the basic principles of flap reconstruction are introduced (Figures 1 to 5). First, areas of laxity adjacent to the defect are identified. Tissue

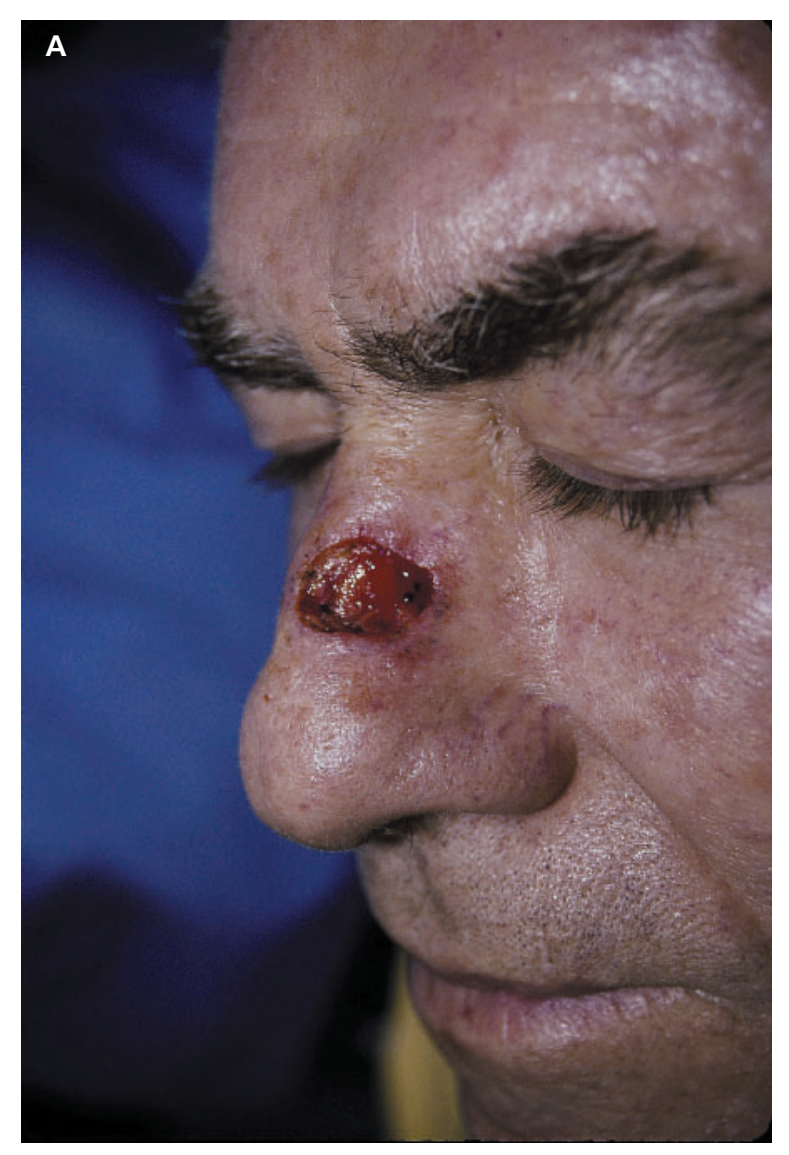

from the same cosmetic unit or subunit provides the optimal match, but skin from an adjacent cosmetic unit is often acceptable. After a tissue reservoir has been defined, the possible mechanisms of tissue movement should be considered and the consequences of moving the tissue evaluated. Tension vectors should avoid free margins and other critical structures. Finally, placing incisions and standing cones within the junction lines between cosmetic units or within relaxed skin tension lines camouflages the appearance of scars. A thorough understanding of the superficial and deep anatomy is a prerequisite for expert flap design.

Although the use of local flaps has many advantages, there are some disadvantages and potential complications that should be considered. First, tissue orientation may be altered by flaps. If the tumor has been inadequately resected, a flap may unnecessarily obscure the surgical site and delay the detection of recurrence. The risks of postoperative bleeding, pain, and infection may be increased by the additional incisions and tissue movement. Flap failure secondary to vascular compromise or infection is increased in patients who are cigarette smokers or those
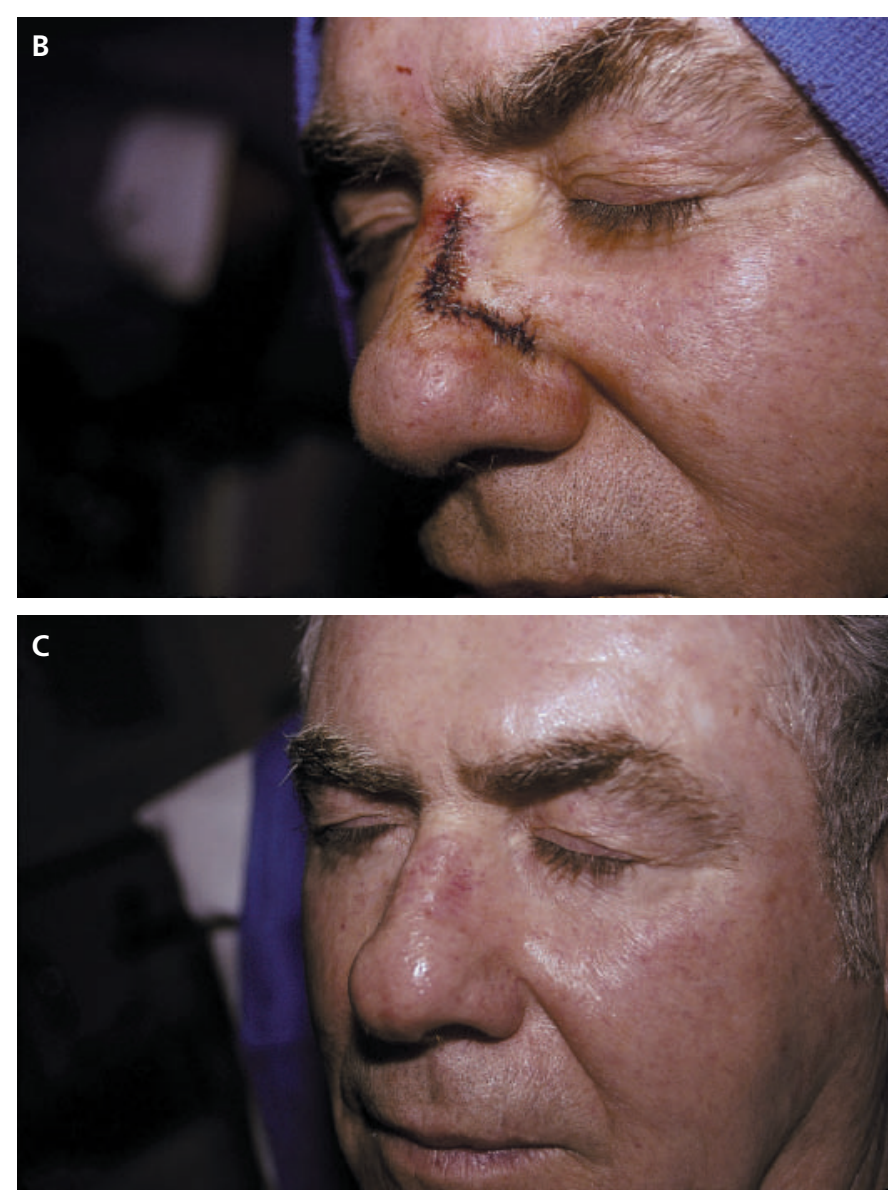

Figure 1. Patient 1. (A) A 65-year-old man had a $1.4 \times 1.0 \mathrm{~cm}$ defect of the left upper nasal sidewall extending to the muscularis following Mohs excision of a basal cell carcinoma. Loose adjacent tissue was identified on the lateral nasal sidewall. (B) A laterally based advancement flap was designed. The principal tension vector resulted from closure of the primary defect side to side, with no distortion of the free margin of the alar rim. The incision lines lie in the lateral ridge of the nose and the alar groove. (C) Result at 7 months. 

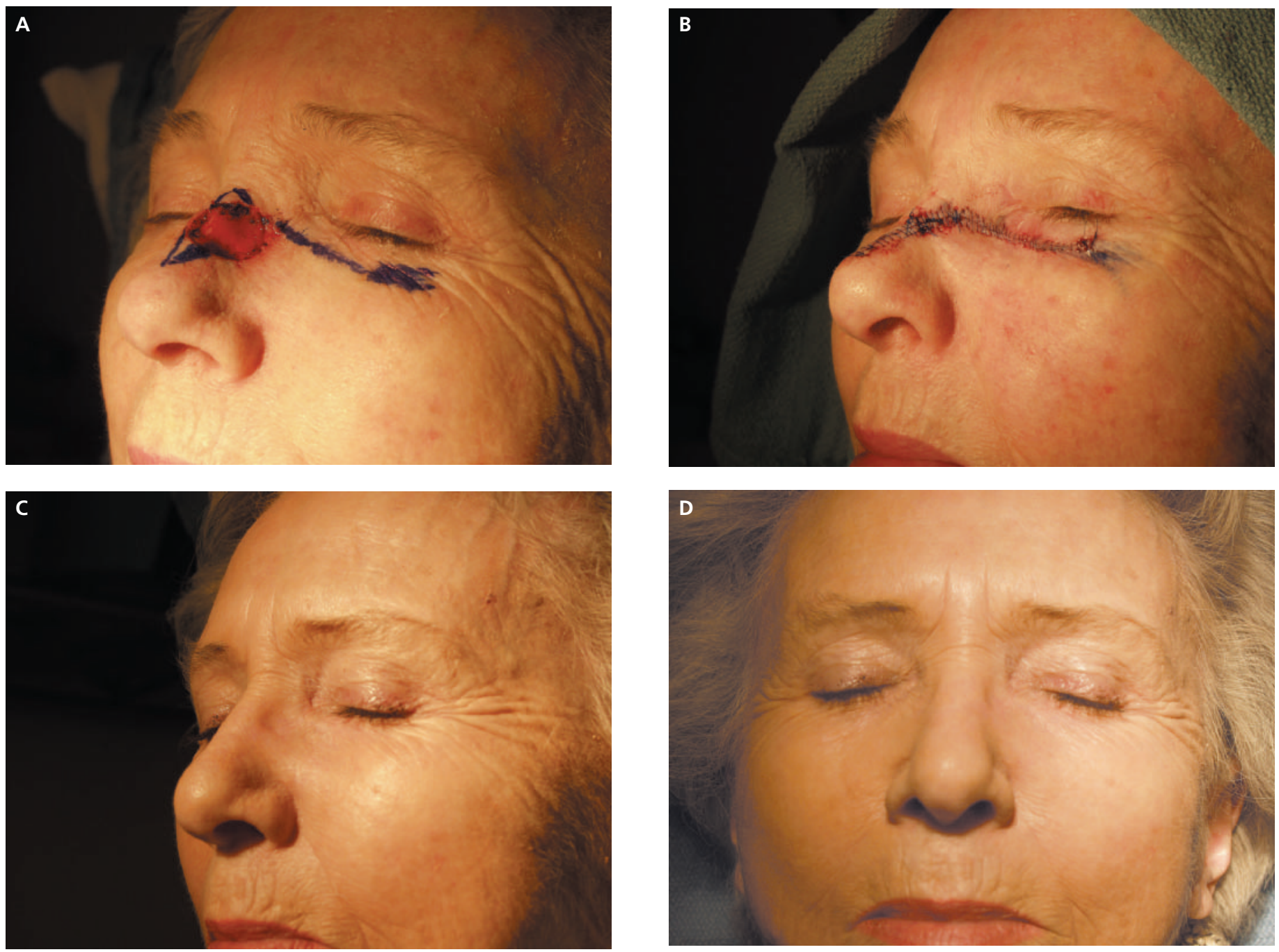

Figure 2. Patient 2. (A) A 79-year-old woman had a $1.4 \times 1.6 \mathrm{~cm}$ defect of the upper left nasal sidewall extending to the periosteum following Mohs excision of a squamous cell carcinoma. Loose adjacent tissue was identified on the lower eyelid and infraorbital cheek. (B) A laterally based advancement flap was designed. The principal tension vector did not distort the free margin of the eyelid. Incision lines lie primarily in the infraorbital crease and periorbital rhytides. (C and D) Result at 7 months, showing no elevated scar or broadening of the nasal root.
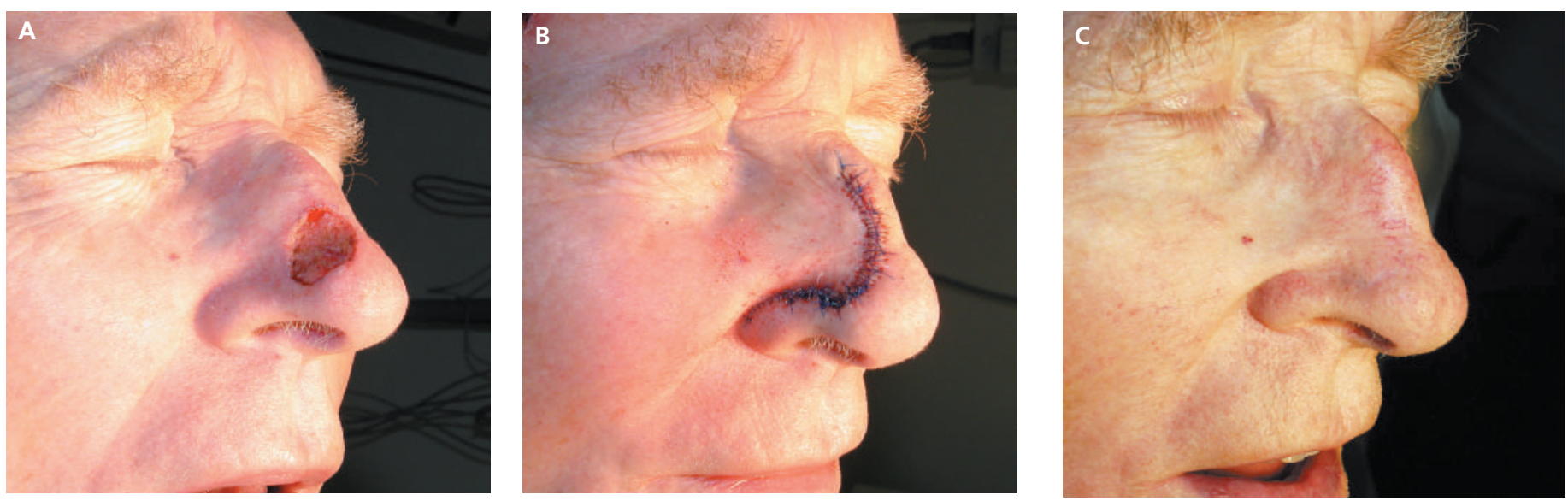

Figure 3. Patient 3. (A) A 79-year-old man had a $1.4 \times 2.0 \mathrm{~cm}$ defect of the right lower nasal sidewall extending to the muscularis following Mohs excision of a basal cell carcinoma. Loose adjacent tissue was identified on the right upper nasal sidewall. (B) A superiorly based rotation flap was designed. The principal tension vector resulted in minimal elevation of the free margin of the alar rim secondary to mucosal edema. The incision lines lie in the lateral ridge of the nose and the alar groove. (C) Result at 8 months, showing no displacement of the alar rim. 

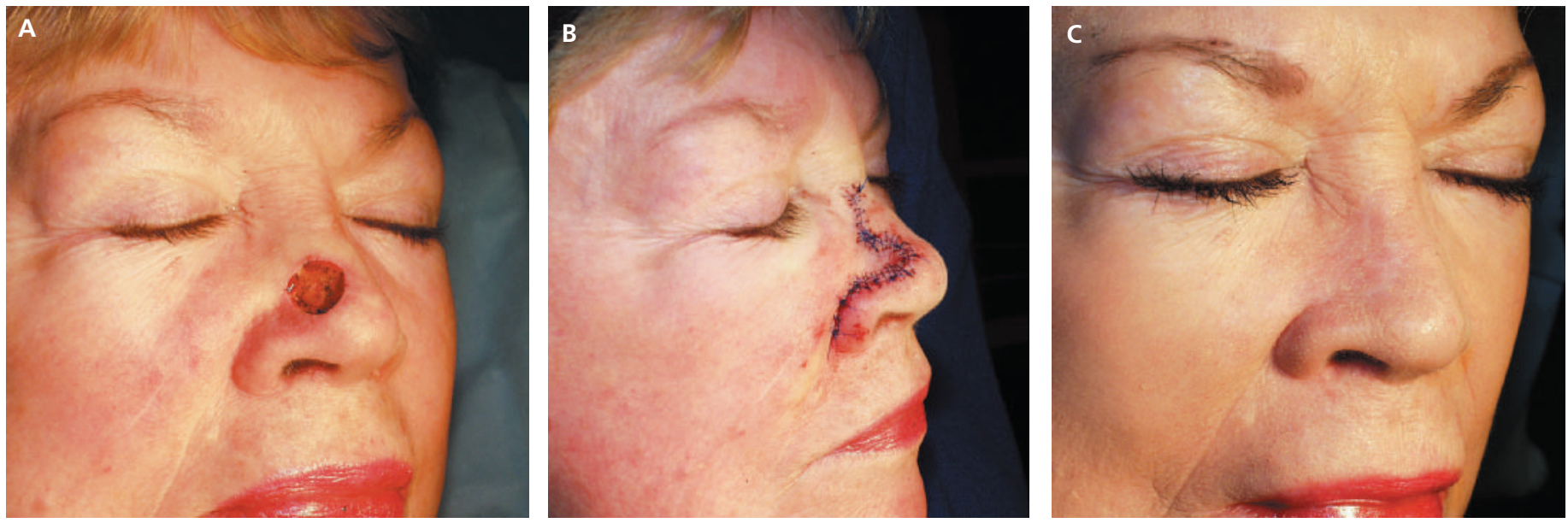

Figure 4. Patient 4. (A) A 79-year-old woman had a $1.3 \times 1.2 \mathrm{~cm}$ defect of the right lower nasal sidewall extending to the deep soft tissue following Mohs excision of a basal cell carcinoma. Loose adjacent tissue was identified on the right upper nasal sidewall. (B) A superiorly based rhombic transposition flap was designed. The principal tension vector resulted in minimal distortion of the free margin of the alar rim. Incision lines lie primarily in the nasofacial sulcus and the alar groove. (C) Result at 8 months, showing no displacement of the alar rim.
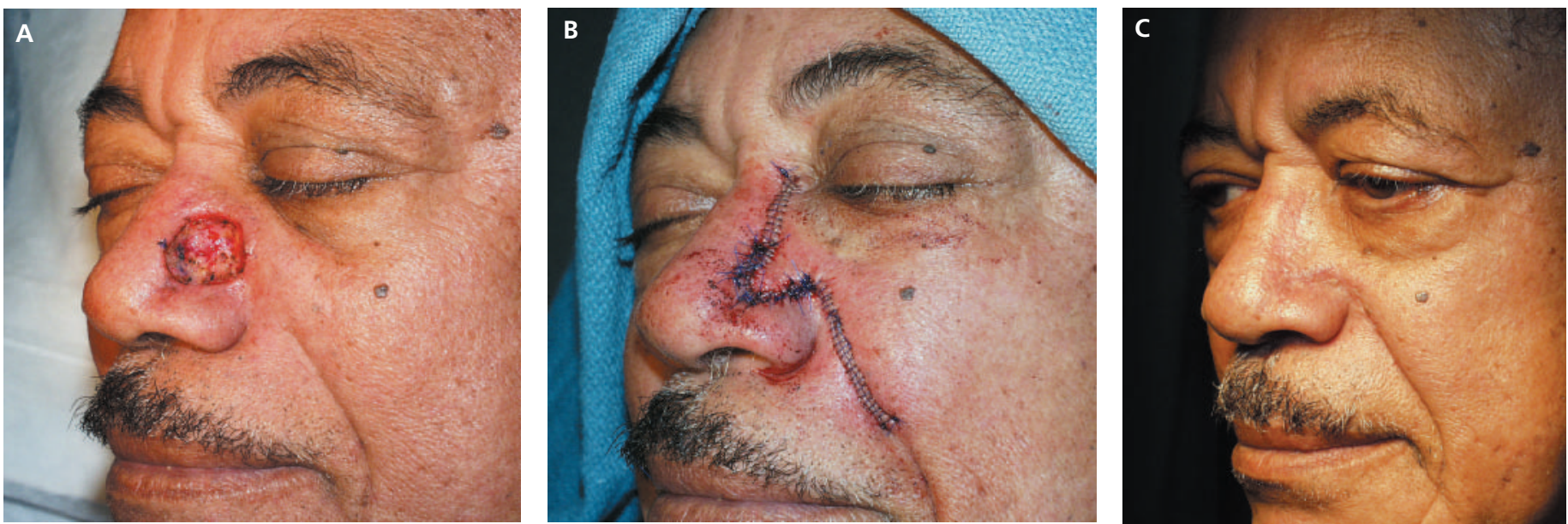

Figure 5. Patient 5. (A) A 64-year-old man had a $1.8 \times 1.7 \mathrm{~cm}$ defect of the left nasal sidewall extending to the deep soft tissue following Mohs excision of a basal cell carcinoma. Loose adjacent tissue was identified on the left medial cheek. (B) A superolaterally based nasolabial transposition flap was designed. The principal tension vector resulted in no distortion of the free margin of the alar rim. Incision lines lie primarily in the nasofacial sulcus, nasolabial fold, and alar groove. (C) Result at 8 months.

with underlying medical problems. ${ }^{8}$ Finally, poorly designed or executed flaps may result in functional impairment or inferior cosmesis.

\section{References}

1. Abide JM, Nahai F, Bennett RG. The meaning of surgical margins. Plast Reconstr Surg 1984;73:492-7.

2. Zitelli JA. Wound healing by secondary intention. J Am Acad Dermatol 1983;9:407-15.

3. Johnson TM, Ratner D, Nelson BR. Soft tissue reconstruction with skin grafting. J Am Acad Dermatol 1992;27:151-65.

4. Summers BK, Siegle RJ. Facial cutaneous reconstructive surgery: general aesthetic principles. J Am Acad Dermatol 1993;29:669-81.

5. Swanson, NA. Classifications, definitions, and concepts in flap surgery. In Cummings CW, editor. Local flaps in facial reconstruction. St. Louis: Mosby; 1995. p. 63-74.

6. Tromovitch TA, Stegman SJ, Glogau RG. Flaps and grafts in dermatologic surgery. Chicago: Year Book; 1989.

7. Johnson TM, Swanson N, Baker SR. Concepts of sliding and lifting tissue movement in flap reconstruction. Dermatol Surg 2000;26:274-8.

8. Goldminz D, Bennett RG. Cigarette smoking and flap and fullthickness graft necrosis. Arch Dermatol 1991;127:1012-5. 